\title{
A new species of Schizopera (Copepoda: Harpacticoida: Miraciidae) from Colombia
}

\author{
Una nueva especie de Schizopera (Copepoda: Harpacticoida: \\ Miraciidae) de Colombia
}

\author{
Juan M. Fuentes-Reinés, ${ }^{1 *}$ M.Sc, Samuel Gómez, ${ }^{2}$ Ph.D.
}

\begin{abstract}
${ }^{1}$ Universidad del Magdalena, Grupo de investigación en Limnología Neotropical. A.A 731 Santa Marta, Magdalena, Colombia. ${ }^{2}$ Universidad Nacional Autónoma de México, Instituto de Ciencias del Mar y Limnología, Unidad Académica Mazatlán, Mazatlán, Sinaloa, México.*Corresponding: juanmanuelfuentesreines@yahoo.com
\end{abstract}

Received: March 2014; Accepted: June 2014.

\begin{abstract}
Objective. The present contribution aims at the description of a new species of the genus Schizopera. Materials and methods. Water samples were collected in littoral areas with mangrove and macrophytes, and in the limnetic zone. Twenty five liters of water were taken. Water samples were filtered with a zooplankton net $(45 \mu \mathrm{m})$ and preserved in $70 \%$ ethanol. The filtered samples were concentrated to $100 \mathrm{ml}$ and examined in a Bogorov camera. Copepods were separated. Observations and drawings of $S$. evelynae sp. nov. were made at a magnification of $1000 X$. Results. Schizopera evelynae sp. nov. seems to be closely related to S. giselae Jiménez -Álvarez 1988 and to S. pratensis Noodt 1958 based on the armature formula of P1-P4, but can be separated from these two species based on the relative length of P1ENP, length/width ratio of P1ENP2, relative length of the outer proximal and distal spines on P4EXP3, shape of the exopod and relative length of the exopodal setae of the female P5, shape and length/width ratio of the male P2ENP2, and male P5 baseoendopodal lobe:exopod length ratio. A key to the species of Schizopera from America is given. Conclusion. A new species of the genus Schizopera is described. The Colombian material shares most characters with S. giselae and S. pratensis.
\end{abstract}

Key words: Copepoda, distribution, harpacticoida, taxonomy, lagoon, new species (Source: CAB).

\section{RESUMEN}

Objetivo. Describir una nueva especie del genero Schizopera. Materiales y métodos. Muestras de agua fueron colectadas en áreas del litoral con mangle y macrófitas, y en la zona limnética. Se tomaron $25 \mathrm{~L}$ de agua. Las muestras de agua fueron filtradas con una red de zooplancton $(45 \mu \mathrm{m})$ y preservadas en etanol al 70\%. Las muestras filtradas se concentraron a $100 \mathrm{ml}$ y fueron analizadas en una cámara de Bogorov. Los copépodos fueron separados. Se realizaron observaciones y dibujos de S. evelynae sp. nov. a un aumento de 1000X. Resultados. Schizopera evelynae sp. nov. parece estar estrechamente relacionada con S. giselae Jiménez-Álvarez 1988 y con S. pratensis Noodt 1958 basado en la fórmula de la armadura de P1-P4, pero puede ser separado de estas dos especies basado 
en la longitud relativa de P1ENP, relación longitud / anchura de P1ENP2, la longitud relativa de las espinas proximal y distal externas en P4EXP3, forma del exópodo y la longitud relativa de las setas del exópodo de la P5 de la hembra, la forma y la relación longitud / anchura del P2ENP2 del macho, y la relación entre la longitud del lóbulo baseoendopodal de la P5 y la longitud del exópodo en el macho. Se presenta una clave para las especies del género Schizopera en América. Conclusiones. Se describe una nueva especie para la ciencia del género Schizopera. Los especímenes colombianos comparten la mayoría de los caracteres con S. giselae y S. pratensis.

Palabras clave: Copepoda, distribución, harpacticoida, taxonomía, laguna, nueva especie (Fuente: CAB).

\section{INTRODUCTION}

Harpacticoida is one of the most important groups of meiobenthos. However, the study of this order from Colombian systems, especially those from estuarine environments, has been largely neglected.

Within Harpacticoida, Miraciidae is one of the most common and abundant families, being composed of about 364 species. Most of the species of Miraciidae are marine living forms inhabiting the benthic realm (1) and are distributed among 53 valid genera (2). Miraciidae contains two large genera, Schizopera (3) and Stenhelia (4) that account for almost $42 \%$ of the whole family. The genus Schizopera comprises about 92 valid species and subspecies worldwide $(5,6), 18$ of which have been described from the Americas (S. tobae tobae (7); S. haitiana(8); S. triacantha (8); S. vicina (9); S. gauldi (10); S. noodti (11); S. knabeni (12); S. californica (12); S. borutzkyi (13); S. anomala (14); S. carolinensis (14); S. tobae cubana (15); S. giselae. (16); S. pori. (16); S. chiloensis (17); S. osana (18); S. hawaiiensis (19); S. costaricana, (20)). Species of genus Schizopera can be found in marine, brackish and freshwater habitats $(21,22)$

Apostolov (23) divided the genus Schizopera into three genera: Schizopera with two subgenera [Schizopera s. str. (23) and Neoschizopera (23)], Eoschizopera (24) with two subgenera [Eoschizopera s. str. (23) and Praeoschizopera (23)], and Schizoperopsis (23) with two subgenera [Schizoperopsis s. str. (23) and Psammoschizoperopsis (23)]. These groupings have been rejected by some authors $(17,20,25)$. Mielke (17) united the genera Eoschizopera and Schizoperopsis into one genus, Schizopera.

The taxonomic position of the genus Schizopera is complex and controversial, and its ecology and phylogenetical relationships have been subject of discussions (17).

Following Mielke $(17,18)$, the subgenera created by Apostolov (23) and the genus Eoschizopera

\section{INTRODUCCIÓN}

Harpacticoida es uno de los grupos más importantes del meiobentos. Sin embargo, el estudio de este orden de copépodos de los sistemas colombianos, especialmente aquéllos de los ambientes estuarios, ha sido muy descuidado.

Dentro de Harpacticoida, Miraciidae es una de las familias más comunes y abundantes, y está compuesta de cerca de 364 especies. La mayoría de las especies de Miraciidae son formas de vida marina que habitan el reino bentónico (1) y están distribuidas en 53 géneros (2). Miraciidae contiene dos grandes géneros: Schizopera (3) y Stenhelia (4) que abarcan cerca del $42 \%$ de toda la familia. El género Schizopera comprende cerca de 92 especies válidas y subespecies en el mundo (5, $6), 18$ de las cuales han sido descritas en ambientes de América (S. tobae tobae (7); S. haitiana(8); S. triacantha(8); S. vicina (9); S. gauldi (10); S. noodti(11); S. knabeni (12); S. californica(12); S. borutzkyi(13); S. anomala (14); S. carolinensis (14); S. tobae cubana (15); S. giselae. (16); S. pori. (16); S. chiloensis (17); S. osana (18); S. hawaiiensis (19); S. costaricana, (20)). Se pueden encontrar especies del género Schizopera en hábitats marinos, salobres y de agua dulce $(21,22)$.

Apostolov (23) dividió el género Schizopera en tres géneros: Schizopera con dos subgéneros [Schizopera s. str. (23) y Neoschizopera(23)], Eoschizopera (24) con dos subgéneros [Eoschizopera s. str. (23) y Praeoschizopera(23)], y Schizoperopsis (23) con dos subgéneros [Schizoperopsis s. str. (23) y Psammoschizoperopsis(23)]. Estas agrupaciones han sido rechazadas por algunos autores $(17,20,25)$. Mielke (17) unificó los géneros Eoschizopera y Schizoperopsis en un solo género: Schizopera.

La posición taxonómica del género Schizopera es compleja y controversial y su ecología y relaciones filogenéticas han sido objeto de discusiones (17).

De acuerdo con Mielke $(17,18)$, el subgénero creado por Apostolov (23) y el género Eoschizopera no deben ser aceptados. Sin embargo, Karanovic 
should not be accepted. Nevertheless, Karanovic (20) challenged Mielke's (17) view. Wells (26) agrees with Karanovic's (20) opinion but considers Schizoperopsis as synonym of Schizopera.

According to Karanovic \& Cooper (5) the subdivision of the genus Schizopera must be now abandoned, being the only exception the genus Eoschizopera $(22,24)$ which includes four species (20). Thus, the generic division proposed by Apostolov (23) has also been abandoned (26).

The description of a new species of Schizopera and an identification key to the species of Schizopera from America is herein presented.

\section{MATERIAL AND METHODS}

Study area. Water samples were collected in Laguna Navío Quebrado (Los Flamencos Fauna and Flora Sanctuary, Camarones, La Guajira Department, Colombia) from April to December 2012 in littoral areas with mangrove and macrophytes, and in the limnetic zone.

Field methods. Twenty five liters of water were taken using a bucket of $25 \mathrm{~L}$. The water samples were filtered with a zooplankton net $(45 \mu \mathrm{m})$ and preserved in $70 \%$ ethanol.

Analytical methods. The filtered samples were concentrated to $100 \mathrm{ml}$ and examined using a Bogorov camera. Copepods were separated and kept in $70 \%$ ethanol. Observations and drawings of $S$. evelynae sp. nov. were made at a magnification of $1000 \mathrm{X}$ from whole and dissected specimens mounted in lactophenol with a Leica compound microscope equipped with phase contrast and a drawing tube.

Museums and terminology. The type material was deposited in the Museo de Colecciones Biológicas de la Universidad del Atlántico Colombia (UARC) and in the Copepoda collection of the Instituto de Ciencias del Mar y Limnología, Mazatlán Marine Station, Sinaloa state, Mexico (EMUCOP).

The terminology proposed by Huys \& Boxshall (27) for the general description was adopted.

Taxonomical account

Family MIRACIIDAE Dana, 1846

Subfamily DIOSACCINAE Sars, 1906

Genus Schizopera Sars, 1905

Schizopera evelynae sp. nov.

(Figures. 1-8)
(20) cuestionó la posición de Mielke (17). Wells (26) está de acuerdo con la opinión de Karanovic (20) pero considera a Schizoperopsis como un sinónimo de Schizopera.

De acuerdo con Karanovic y Cooper (5) la subdivisión del género Schizopera debe ser abandonada, siendo la única excepción el género Eoschizopera $(22,24)$ que incluye cuatro especies (20). Así, la división genérica propuesta por Apostolov (23) también debe ser abandonada (26).

Se presenta aquí la descripción de una nueva especie y una clave de identificación de las especies de Schizopera de América.

\section{MATERIALES Y MÉTODOS}

Área de Estudio. Se recolectaron muestras de agua en la Laguna Navío-Quebrado (Santuario de Fauna y Flora "Los Flamencos", Camarones, Departamento de la Guajira, Colombia) entre abril y diciembre de 2012 en áreas del litoral con manglares y macrófitas y en la zona limnética.

Métodos de campo. Se tomaron veinticinco litros de agua, utilizando un balde de 25 L. Las muestras de agua fueron filtradas con una red de zooplanton (45 $\mu \mathrm{m})$ y conservadas en etanol al $70 \%$.

Métodos analíticos. Se concentraron las muestras filtradas en un volúmen final de 100 $\mathrm{ml}$ y se analizaron utilizando una cámara de Bogorov. Se separaron los copépodos y se conservaron en etanol al $70 \%$. Se hicieron observaciones y dibujos a partir de individuos de $S$. evelynae sp. nov., completos y disectados montados en lactofenol a un aumento máximo de 1000X con un microscopio compuesto Leica equipado con contraste de fases y tubo de dibujo.

Museos y terminología. El material tipo fue depositado en el Museo de Colecciones Biológicas de la Universidad del Atlántico - Colombia (UARC) y en la colección de Copépodos del Instituto de Ciencias del Mar y Limnología, de la Unidad Académica Mazatlán en Sinaloa, México (EMUCOP).

Se adoptó la terminología propuesta por Huys \& Boxshall (27) para la descripción general.

Registro taxonómico

Familia MIRACIIDAE Dana, 1846

Sub-familia DIOSACCINAE Sars, 1906

Género SchizoperaSars, 1905

Schizopera evelynae sp. nov.

(Figuras 1-8) 
Type material.One female holotype (UARC154M), one male allotype (UARC155M), and 16 female and 14 male paratypes (UARC156M) preserved in alcohol, and 29 female and 30 male paratypes preserved in alcohol (EMUCOP 0412-01) and 2 female (EMUCOP 0412-02, EMUCOP 0412-03) and 2 male (EMUCOP 0412-04, EMUCOP 0412-05) dissected paratypes; April to December, 2012. Leg. Juan M. Fuentes Reinés.

Type locality. Laguna de Navío Quebrado, Los Flamencos Fauna and Flora Sanctuary, Camarones, La Guajira Department, Colombia $\left(11^{\circ} 25^{\prime} \mathrm{N}, 7^{\circ} 5^{\prime} \mathrm{W}\right)$. Salinity, from 0-28 PSU. Schizopera evelynae sp. nov. was found in the limnetic zone and in vegetated areas (mangroves); salinity, 28 PSU.

Etymology. The species is named in honor to Dr Evelyn Zoppy de Roa, for her work on zooplankton from Venezuelan systems and for her legacy and leadership of new generations of planktologists.

Description of female. Habitus (not shown) fusiform, tapering from the posterior part of the cephalothorax to the posterior part of the body. Total body length, measured from the tip of rostrum to the posterior margin of caudal rami, ranging from $532 \mu \mathrm{m}$ to $602 \mu \mathrm{m}(\mathrm{n}=11$, mean $=567 \mu \mathrm{m})$. First urosomite (P5-bearing somite) without, posterior margin of genital double-somite, fourth and fifth urosomites with posterior finely serrated hyaline frill; dorsal
Material tipo. Un holotipo hembra (UARC154M), un alotipo macho (UARC155M), y 16 paratipos hembra y 14 machos (UARC156M) preservados en alcohol, y 29 paratipos hembra y 30 machos preservados en alcohol (EMUCO 0412-01) y 2 paratipos disectados hembras (EMUCOP 0412-02, EMUCOP 0412-03) y 2 machos (EMUCOP 0412-04, EMUCOP 0412-05); Abril a diciembre de 2012. Leg. Juan M. Fuentes Reinés.

Localidad tipo. Laguna de Navío Quebrado, Santuario de Flora y Fauna "Los Flamencos", Camarones, Departamento de la Guajira, Colombia $\left(11^{\circ} 25^{\prime} \mathrm{N}, 75^{\circ} 5^{\prime} \mathrm{W}\right)$. Salinidad de 0-28 PSU. Schizopera evelynae sp. nov. fue hallada en la zona limnética y en la zona cubierta por vegetación (manglares); salinidad, 28 PSU.

Etimología. La especie fue nombrada en honor a la Dra. Evelyn Zoppy de Roa, por su trabajo en el zooplancton de sistemas venezolanos y por su legado y liderazgo de nuevas generaciones de zooplanctólogos.

Descripción de la hembra. Habitus fusiforme (no se muestra), estrechándose desde la parte posterior del cefalotórax a la parte posterior del cuerpo. Longitud total del cuerpo, medido desde la punta de rostro hasta el margen posterior de las ramas caudales, desde $532 \mu \mathrm{m}$ hasta 602 $\mu \mathrm{m}(\mathrm{n}=11$, media $=567 \mu \mathrm{m})$. Primer urosomita (aquel que lleva la P5), sin membrana hialina posterior, margen posterior del somita genital doble, cuarto y quinto urosomitas con membrana hialina posterior finamente aserrada; membrana

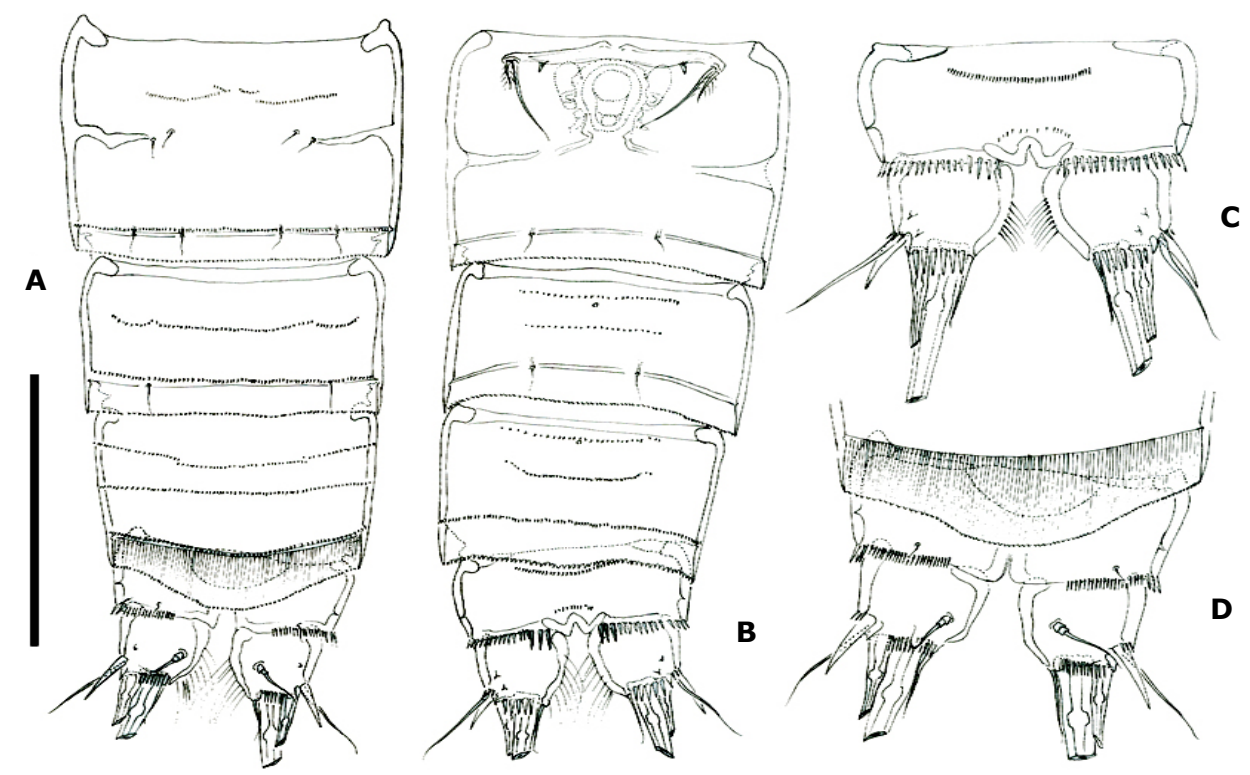

Figure 1. Schizopera evelynae sp. nov., female, paratype (EMUCOP 0412-02). A, urosome, dorsal, P5-bearing somite omitted; $\mathrm{B}$, urosome, ventral, showing P6, P5 bearing-somite omitted. C, posterior part of fifth urosomite, anal somite and caudal rami, dorsal; D, anal somite and caudal rami, ventral. Scale bar: A, B, $100 \mu \mathrm{m} ; \mathrm{C}, \mathrm{D}, 140 \mu \mathrm{m}$. 


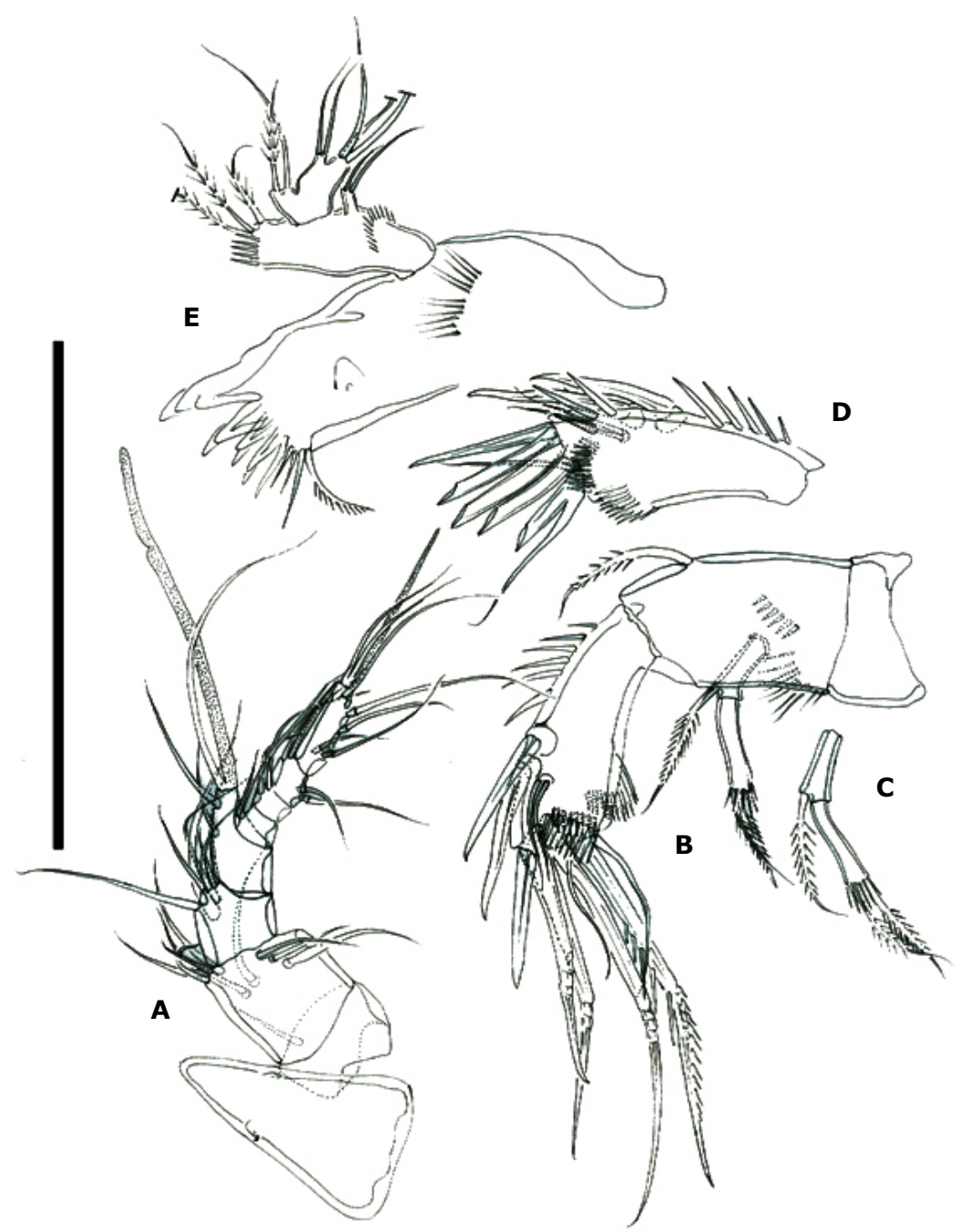

Figure 2. Schizopera evelynae sp. nov., female. A, antennule and rostrum, dorsal; B, antenna; $C$, exopod of antenna, another view; $D$, free endopodal segment of antenna, another view; $E$, mandible. $A, E$, paratype EMUCOP 0412-02; B-D, paratype EMUCOP 0412-03. Scale bar: A, $100 \mu \mathrm{m}$; B-E, $70 \mu \mathrm{m}$.

hyaline frill of fifth urosomite bulging medially (Figures 1A). Genital double-somite fused dorsally and ventrally, with lateral rib indicating former division (Figure 1A, B); ventral surface plain, bearing P6, the latter represented by 1 outer short plumose seta and 1 inner long slender element (Figure 1B). Dorsal and ventral surface of genital double-somite, fourth and fifth urosomite with spinular pattern as figured (Figure $1 A, B)$. Anal somite with semicircular smooth operculum (Figures 1A, C); with ventral spinules as shown (Figure 1D). Caudal rami as long as wide (Figures 1A-D); with 6 elements.

Rostrum not fused to cephalothorax, elongate, triangular, reaching tip of the second antennular segment (Figure $2 \mathrm{~A}$ ). hialina dorsal del quinto urosomita extendido en la parte media (Figuras 1A). El somita genital doble fusionado dorsalmente y ventralmente, con restos de división dorsalmente y lateralmente indicando división previa (Figura 1A, B); superficie ventral sin ornamentación, con $\mathrm{P} 6$, esta última representada por una seta externa plumosa y un elemento interno largo y delgado (Figura 1B), superficie dorsal y ventral del somita genital doble, cuarto y quinto urosomita con espínulas según se muestra (Figura 1A, B). Somita anal con opérculo semicircular, sin ornamentación (Figuras $1 A, C$ ); con espínulas ventrales como se muestra (Figura 1D), ramas caudales tan largas como anchas (Figuras $1 \mathrm{~A}-\mathrm{D})$; con 6 elementos.

Rostro no fusionado con el cefalotórax, alargado, triangular, alcanzando el margen dista del segundo segmento antenular (Figura 2A). 
Antennule (Figure 2A) 8-segmented; armature formula: 1(1), 2(9), 3(7), 4(2+(1+ae)), 5(2), 6(3), 7(4), 8(5+acrotek).

Antenna (Figure 2B-D). Allobasis with short rows of spinules along inner margin proximally and at base of exopod, with 1 abexopodal seta. Exopod 2-segmented; first segment with 1 slender plumose seta; second segment with 2 setae. Free endopodal segment with inner row of spinules, and armed with 2 lateral inner spines and 2 slender setae, and with seven distal elements ( 1 spine, 3 geniculate elements, 1 slender seta, and 1 strong spinulose element fused to 1 slender setae basally).

Mandible (Figure 2E). Gnathobasis with bidentate pars incisiva, three spinules and 1 pinnate seta. Coxa-basis with 3 plumose setae distally. Exopod small, with 2 setae. Endopod much larger than exopod, 1-segmented, with 2 inner setae (one of them shorter), and 5 distal setae.
Anténula (Figura 2A) con 8 segmentos; fórmula de de la armadura: 1(1), 2(9), 3(7), 4(2+(1+ae)), 5(2), 6(3), 7(4), 8(5+acrotek).

Antena (Figura 2B-D). Alobase con filas cortas de espínulas a lo largo del margen interno proximal y en la base de exópodo, con una seta abexopodal. Exópodo de 2 segmentos; primer segmento con una seta plumosa delgada; segundo segmento con dos setas. Segmento libre del endópodo con una fila interna de espínulas, y armado con dos espinas internas laterales y 2 setas delgadas, y con siete elementos distales ( 1 espina, 3 elementos geniculados, 1 seta delgada, y 1 elemento fuerte espinulado fusionado basalmente a 1 seta fina).

Mandíbula (Figura 2E). Gnatobase con incisivos pareados, tres espínulas y 1 seta pinada. Coxabase con 3 setas plumosas distales. Exópodo pequeño, con dos setas. Endópodo mucho más largo que el exópodo, con 1 segmento, con dos setas internas (una de ellas más corta), y 5 setas distales.

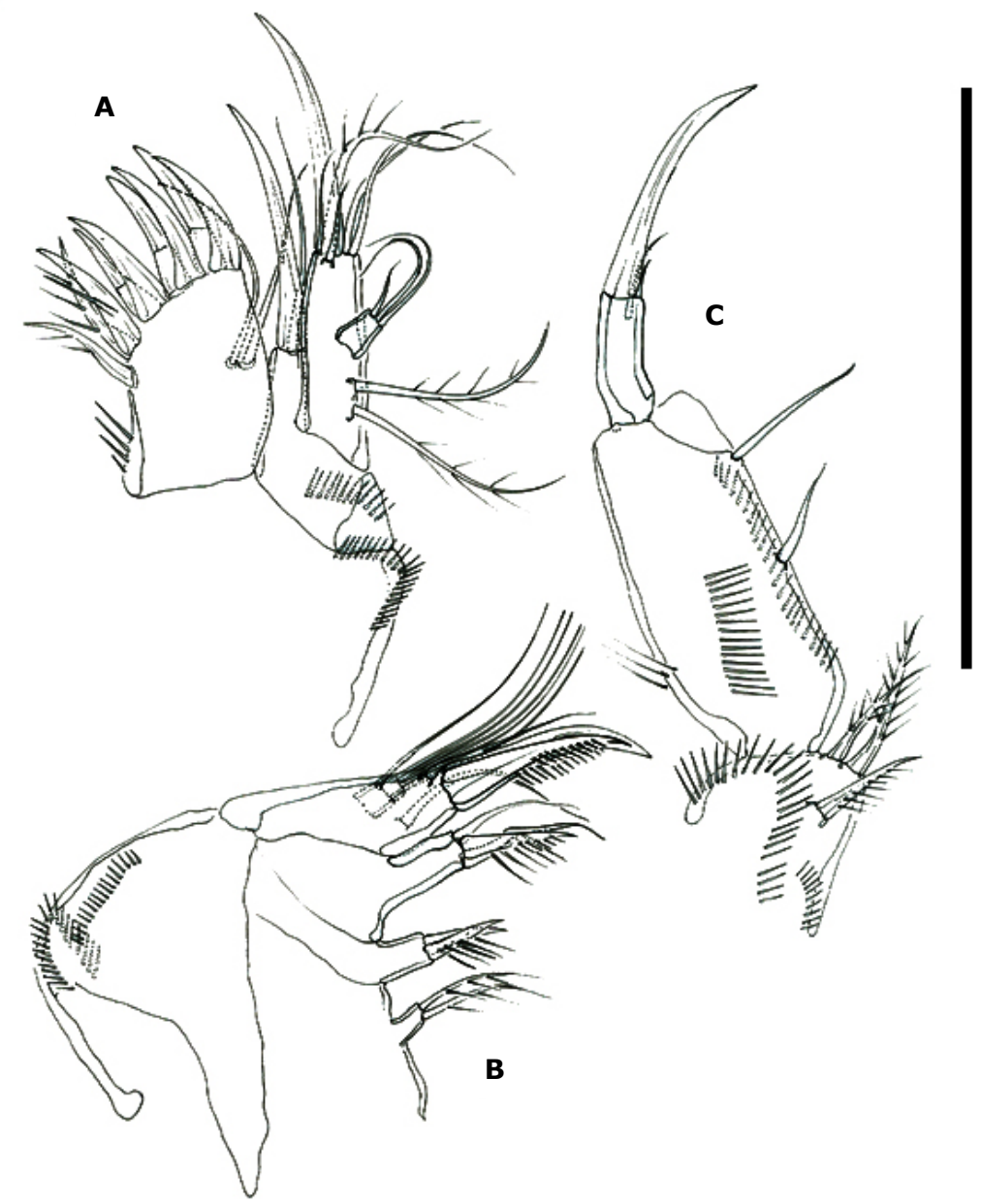

Figure 3. Schizopera evelynae sp. nov., female. A, maxillule; B, maxilla; C, maxilliped. A-C, paratype EMUCOP 0412-02. Scale bar: $50 \mu \mathrm{m}$. 
Maxillule (Figure 3A). Praecoxal arthrite with 8 strong spines, 1 pinnate lateral element and 2 surface setae. Coxa with 1 strong and 1 slender seta. Basis with 6 elements. Exopod fused to basis, with 2 setae. Endopod 1 - segmented, with 3 slender setae.

Maxilla (Figure 3B). Syncoxa with three endites; first and second endites with 2 elements each, third endite with 3 elements. Endopod 2-segmented, with 6 setae.

Maxilliped (Figure 3C). Basis with spinules as shown and armed with 1 subdistal and 2 apical pinnate setae. Endopod 2-segmented; first segment with row of spinules along inner margin and 2 setae; second segment with strong claw and 2 accompanying setae (1 of them very short).
Maxílula (Figura 3A). Artrito precoxal con 8 espinas fuertes, 1 elemento lateral pinado y 2 setas superficiales. Coxa con una seta fuerte y 1 seta delgada. Base con 6 elementos. Exópodo fusionado a la base, con 2 setas. Endópodo con 1 segmento, con 3 setas delgadas.

Maxila (Figura 3B). Syncoxa con tres enditos; primer y segundo enditos con 2 elementos cada uno, tercer endito con 3 elementos. Endópodo con 2 segmentos, con 6 setas

Maxilípedo (Figura 3C). Base con espínulas como se muestra y armado con 1 seta subdistal y 2 setas apicales pinadas. Endópodo con 2 segmentos; primer segmento con fila de espínulas a lo largo del margen interno y con dos setas; segundo segmento con garra fuerte y dos setas (1 de ellas muy corta).

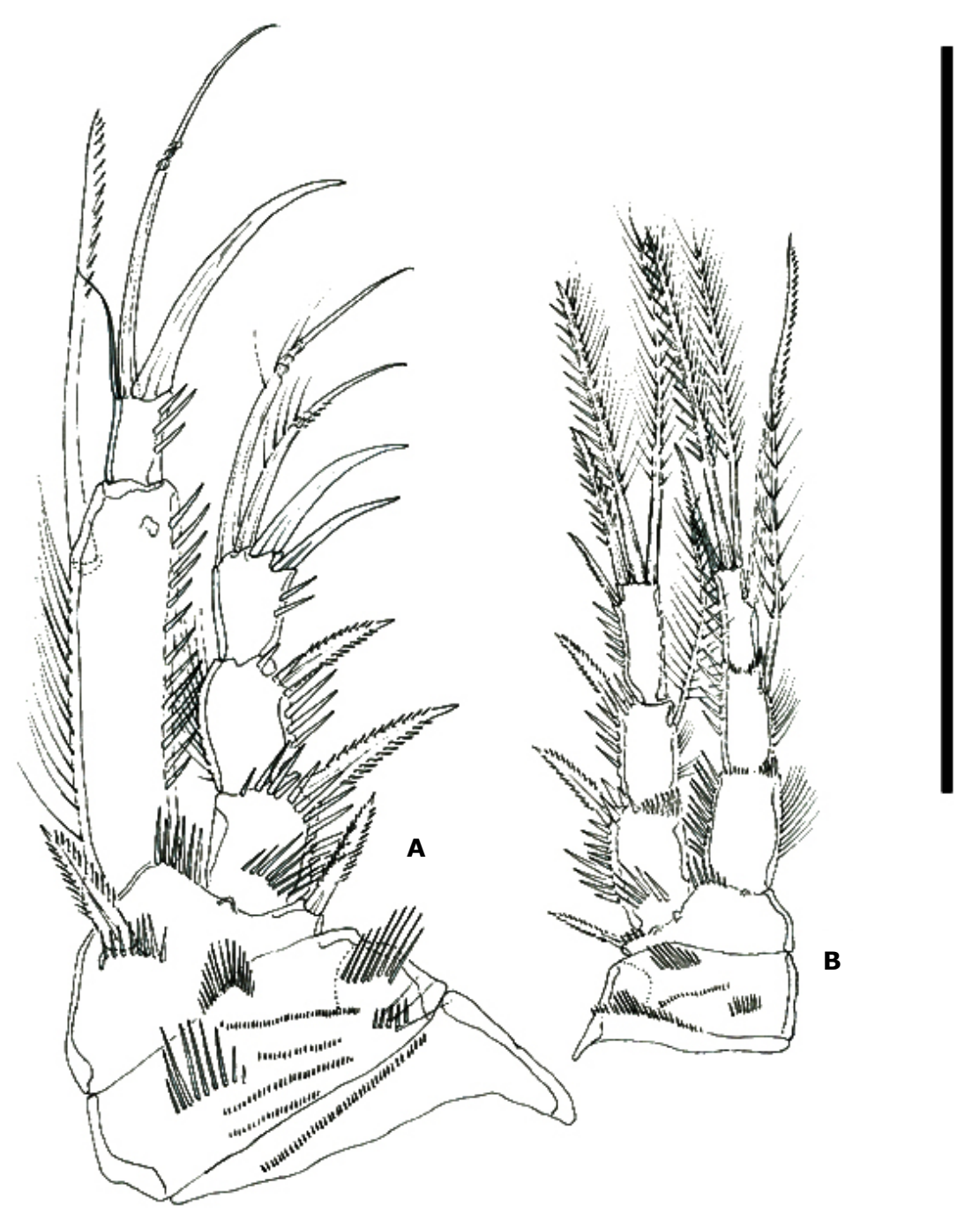

Figure 4. Schizopera evelynae sp. nov., female. $A, P 1$, anterior; $B, P 2$, anterior. $A$, paratype EMUCOP 0412-02; B, paratype EMUCOP 0412-03. Scale bar: A, $100 \mu \mathrm{M} ; \mathrm{B}, 124 \mu \mathrm{m}$. 
P1 (Figure 4A). Praecoxa, coxa and basis ornamented as shown. Basis with inner and outer spines. Exopod 3-segmented; exopodal segments without inner armature; EXP 3 with 4 elements. Endopod 2-segmented; first segment longer than exopod, about 4 times as long as wide, with a long inner seta subdistally; second segment about 1.6 times as long as wide, with one strong spine, one geniculate element and one slender seta.

P2 (Figure 4B). Praecoxa, coxa and basis ornamented as figured. Basis with outer spiniform element. Rami 3-segmented. Exopod as long as endopod; EXP 1 and 3 without, EXP 2 with inner seta; EXP 3 with 4 elements. First endopodal segment without, second segment with 1 inner and 3 apical elements.

P3 (Figure 5). Spinular ornamentation of praecoxa, coxa and basis as shown. Basis with outer seta. Rami 3-segmented. Exopod as long as endopod; EXP 1 and 3 without, EXP 2 with inner seta, EXP 3 with 4 elements in all. ENP 1-2 with inner seta; ENP 3 with 1 strong inner seta and 3 apical elements.
P1 (Figura 4A). Precoxa, coxa y base ornamentadas como se muestra. Base con espina interna y externa. Exópodo con 3 segmentos; segmentos del exópodo sin armadura interna; EXP 3 con 4 elementos. Endópodo con 2 segmentos; primer segmento más largo que el exópodo, cerca de 4 veces más largo que ancho, con una seta interna larga subdistal; el segundo segmento cerca de 1.6 veces más largo que ancho, con una espina fuerte, un elemento geniculado y una seta fina.

P2 (Figura 4B).Precoxa, coxa y base ornamentadas como se muestra. Base con elemento externo espinoforme. Ramas con 3 segmentos. Exópodo tan largo como el endópodo; EXP 1 y 3 sin, EXP 2 con seta interna; EXP 3 con 4 elementos. Primer segmento endopodal sin, segundo segmento con 1 elemento interno; tercer segmento con 3 elementos..

P3 (Figura 5).Con espínulas en precoxa, coxa y base como se muestra. Base con seta externa. Ramas con 3 segmentos. Exópodo tan largo como el endópodo; EXP 1 y 3 sin, EXP 2 con seta interna, EXP 3 con 4 elementos en total. ENP 1-2 con seta interna; ENP 3 con una seta interna fuerte y 3 elementos apicales.

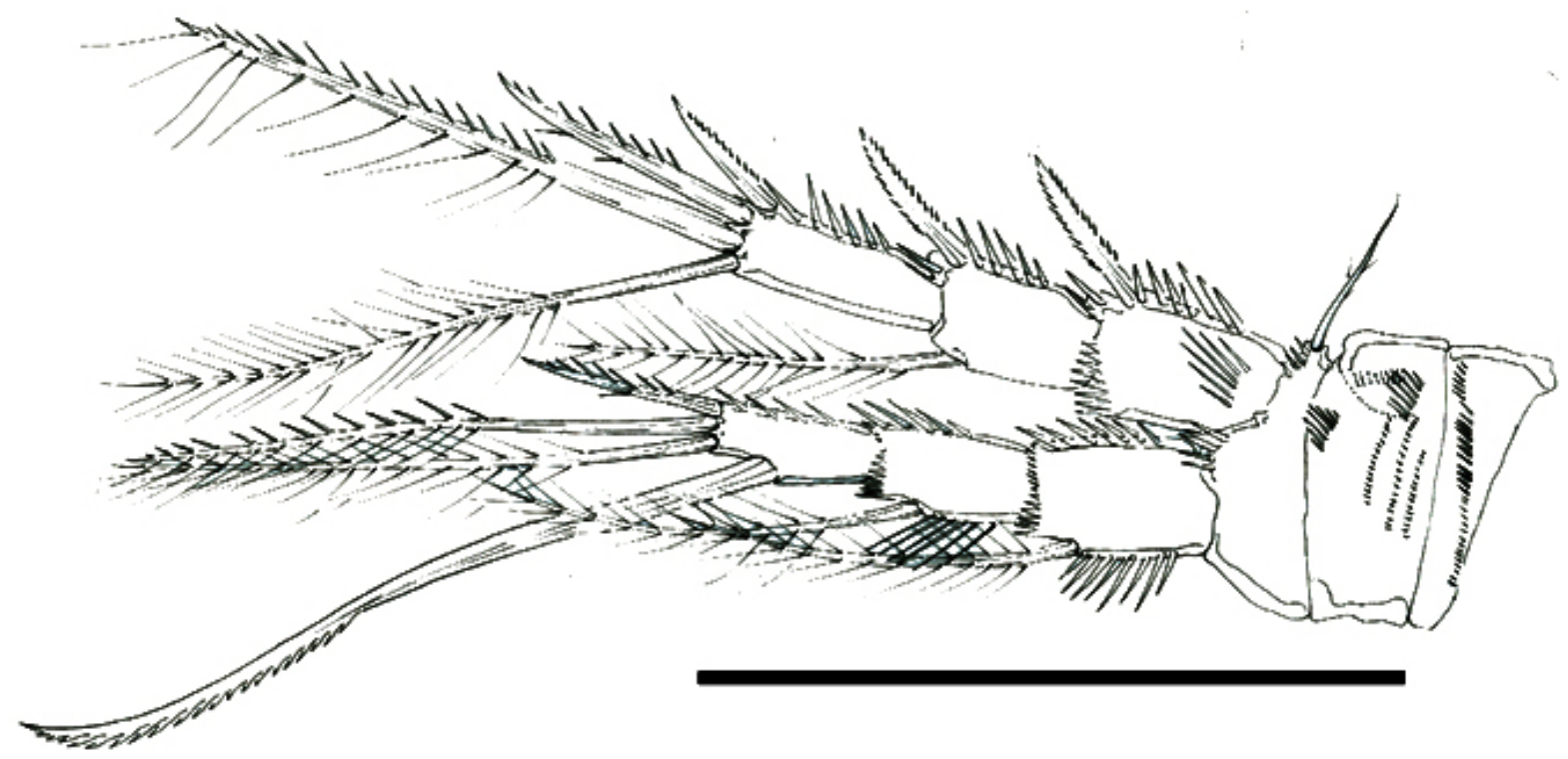

Figure 5. Schizopera evelynae sp. nov., female. P3, anterior. A, paratype EMUCOP 0412-02. Scale bar: $100 \mu \mathrm{m}$.

P4 (Figure 6A). Praecoxa, coxa and basis as in P3. Rami 3-segmented. Exopod noticeably longer than endopod; EXP 1-3 without, EXP 2 with inner seta; EXP 3 with 4 elements in all.

P5 (Figure 6B). Baseoendopodal lobe with 2 inner and 2 distal setae plus outer seta of basis. Exopod reaching beyond baseoendopod, with 6 setae.
P4 (Figura 6A).Precoxa, coxa y base como en P3. Ramas con 3 segmentos. Exópodo claramente más largo que el endópodo; EXP 1-3 sin, EXP 2 con seta interna; EXP 3 con 4 elementos en total.

P5 (Figura 6B). Lóbulo del basoendópodo con dos setas internas y 2 distales, y seta externa de la base. El exópodo alcanza más allá del basoendópodo, con 6 setas. 
Armature formula P1-P4 as follows:

$\begin{array}{lll} & \text { Exopod } & \text { Endopod } \\ \text { P1 } & \text { I-0; I-0; I,I1,1 } & 0-1 ; \text { I11 } \\ \text { P2 } & \text { I-0; I-1;I,I1,1 } & 0-0 ; 0-1 ; \mathrm{I}, 2,1 \\ \text { P3 } & \text { I-0;I-1;I,I1,1 } & 0-1 ; 0-1 ; \mathrm{I}, 2,1 \\ \text { P4 } & \text { I-0;I-1;I,I1,1 } & 0-1 ; 0-1 ; \mathrm{I}, 2,0\end{array}$

Fórmula de setas y espinas de P1-P4 como sigue:

$\begin{array}{lll} & \text { Exópodito } & \text { Endópodito } \\ \text { P1 } & \text { I-0; I-0; I,I1,1 } & 0-1 ; \mathrm{I} 11 \\ \text { P2 } & \text { I-0; I-1;I,I1,1 } & 0-0 ; 0-1 ; \mathrm{I}, 2,1 \\ \text { P3 } & \text { I-0;I-1;I,I1,1 } & 0-1 ; 0-1 ; \mathrm{I}, 2,1 \\ \text { P4 } & \text { I-0;I-1;I,I1,1 } & 0-1 ; 0-1 ; \mathrm{I}, 2,0\end{array}$

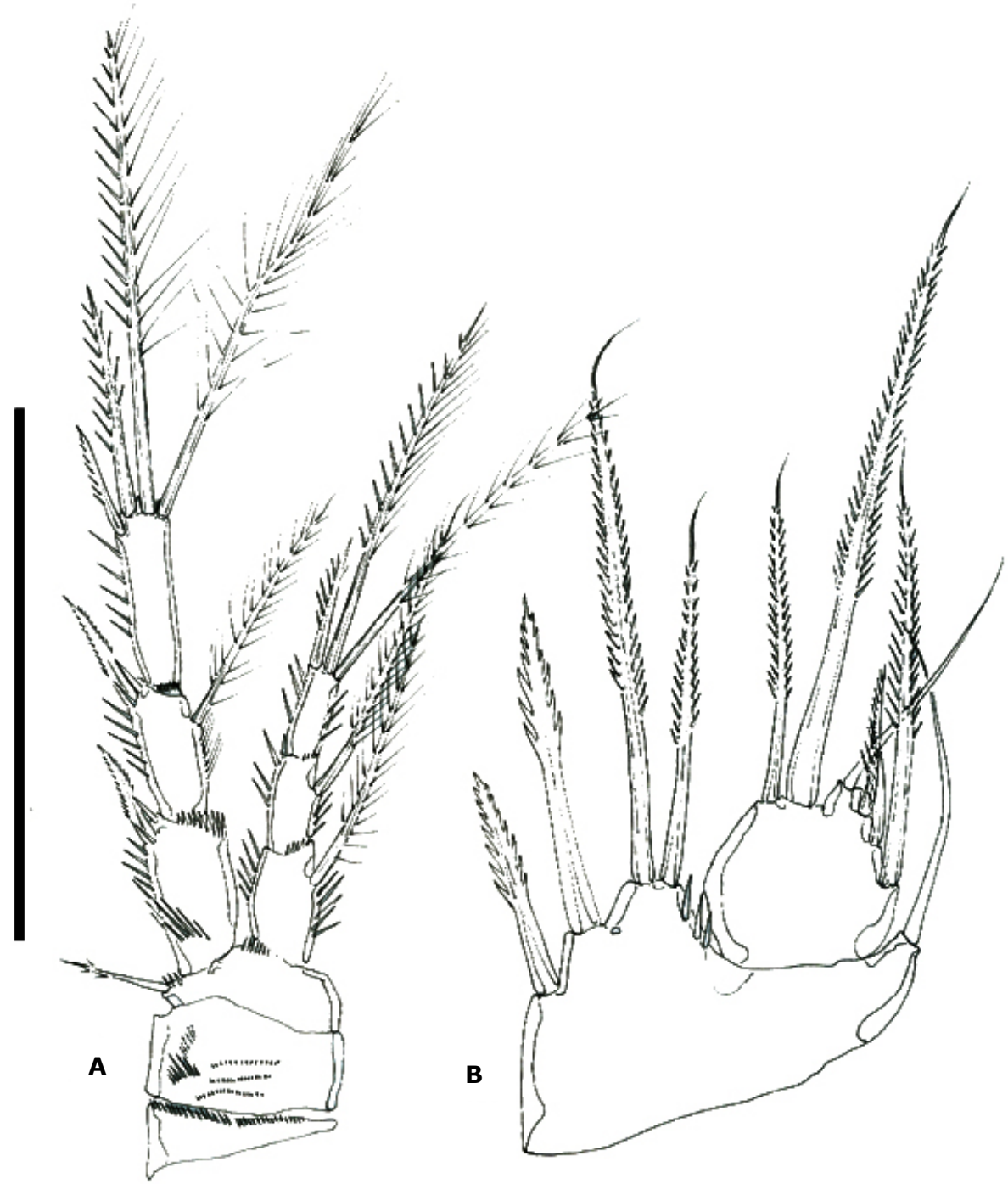

Figure 6. Schizopera evelynae sp. nov., female. A, P4, anterior; B, P5, anterior. A, B, paratype EMUCOP 0412-02. Scale bar: $A, 100 \mu \mathrm{m} ; \mathrm{B}, 70 \mu \mathrm{m}$

Description of male. Habitus and rostrum (not shown) as in female. Total body length, measured from tip of rostrum to posterior margin of caudal rami, ranging from 400 to $420 \mu \mathrm{m}$ $(n=11 ;$ mean $=410)$. Dorsal and ventral spinular ornamentation of urosomites (Figure 7A, B) as shown. Anal somite and caudal rami as in female.
Descripción del macho. Habitus y rostro (no se muestran) como en la hembra. Longitud total del cuerpo, medido desde la punta de rostro al margen posterior de las ramas caudales, desde $400 \mu \mathrm{m}$ hasta $420 \mu \mathrm{m}(\mathrm{n}=11$; media $=410)$. Con espínulas dorsales y ventrales de los urosomitas (Figura 7A, B) como se muestra. Somita anal y ramas caudales como en la hembra. 


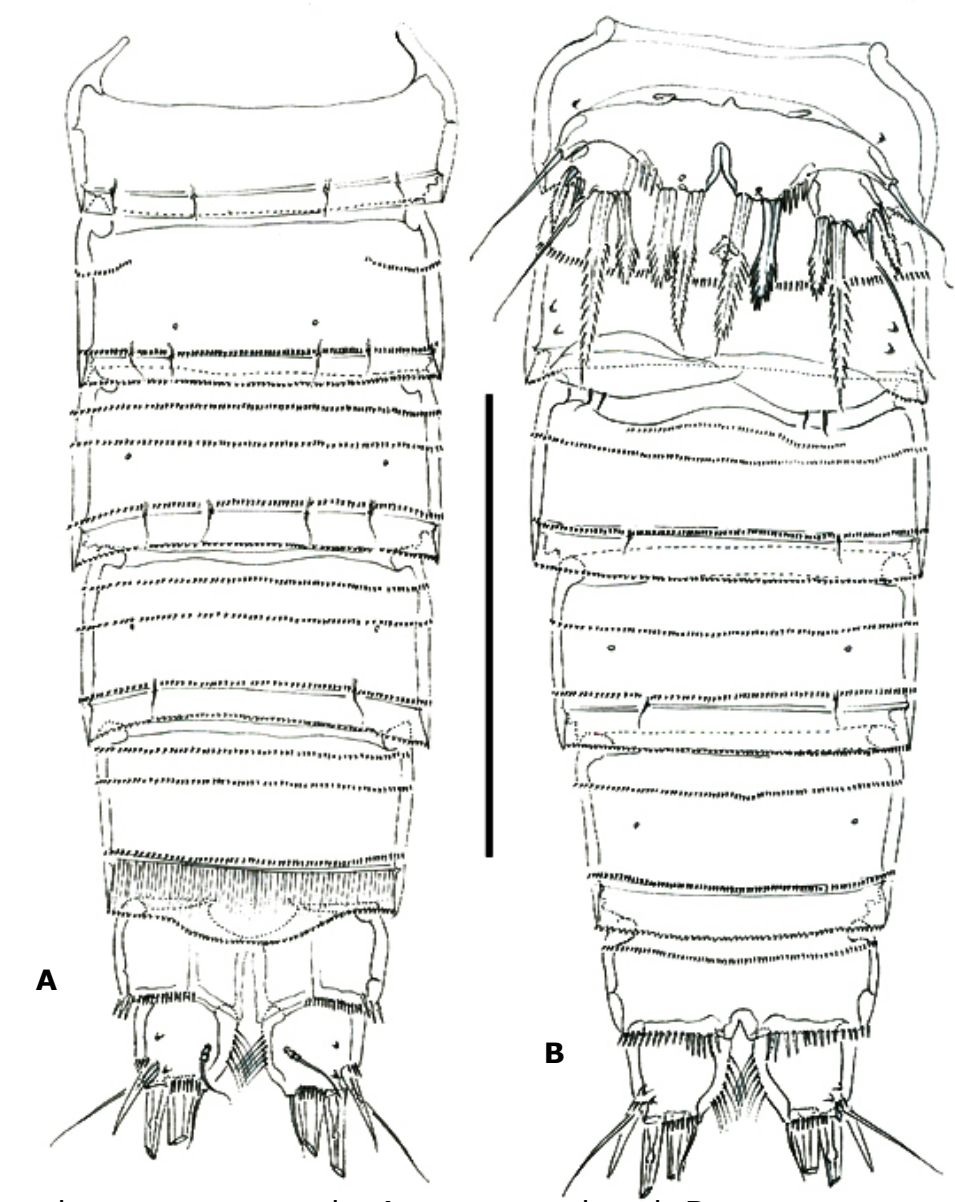

Figure 7. Schizopera evelynae sp. nov., male. A, urosome, dorsal; B, urosome, ventral, showing P5 and P6. A, B, paratype EMUCOP 0412-04. Scale bar: $100 \mu \mathrm{m}$.

Antennule (Figure 8A) 8-segmented, haplocer. First and fifth segment with spinules, second segment wide, third segment narrow, fourth segment swollen. Armature formula difficult to define, but most probably as follows: $1(1)-2(9)-3(6)-4(7+(1+a e))-5(2)-6(1)-7(4)-$ $8(4+$ acrothek $)$.

Antenna, mandible, maxillule, maxilla and maxilliped as in female.

P1 as in female except for dimorphic inner process of basis (Figure $8 \mathrm{~B}$ ).

P2 EXP (not shown) as in female. Endopod (Figure 8C) 2-segmeted, proximal segment without armature, distal segment modified as in figures 8D, E.

P3 as in female, except for the presence of a characteristic inner flattened hyaline spine on the third exopodal segment (Figure 8F).

P4 (not illustrated) as in female.
Anténula (Figura 8A) con 8 segmentos, haplócera. Primer y quinto segmentos con espínulas. Segundo segmento ancho, tercer segmento angosto, cuarto segmento goloboso. Fórmula de setas difícil de definir, pero lo más probable es como sigue: $1(1)-2(9)-3(6)-4(7+(1+a e))-5(2)-6(1)-7(4)-$ $8(4+$ acrothek $)$.

Antena, mandíbula, maxílula, maxila y maxilípedo como en la hembra.

P1 como en la hembra excepto por proceso interno dimórfico en la base (Figura 8B).

P2 EXP (no se muestra) como en la hembra. Endópodo (Figura 8C) con 2 segmentos, segmento proximal sin armadura, segmento distal modificado como en las Figuras 8D, E.

P3 como en la hembra, excepto por la presencia de una espina interna plana hialina característica en el tercer segmento del exópodo (Figura 8F).

P4 (sin ilustrar) como en la hembra. 


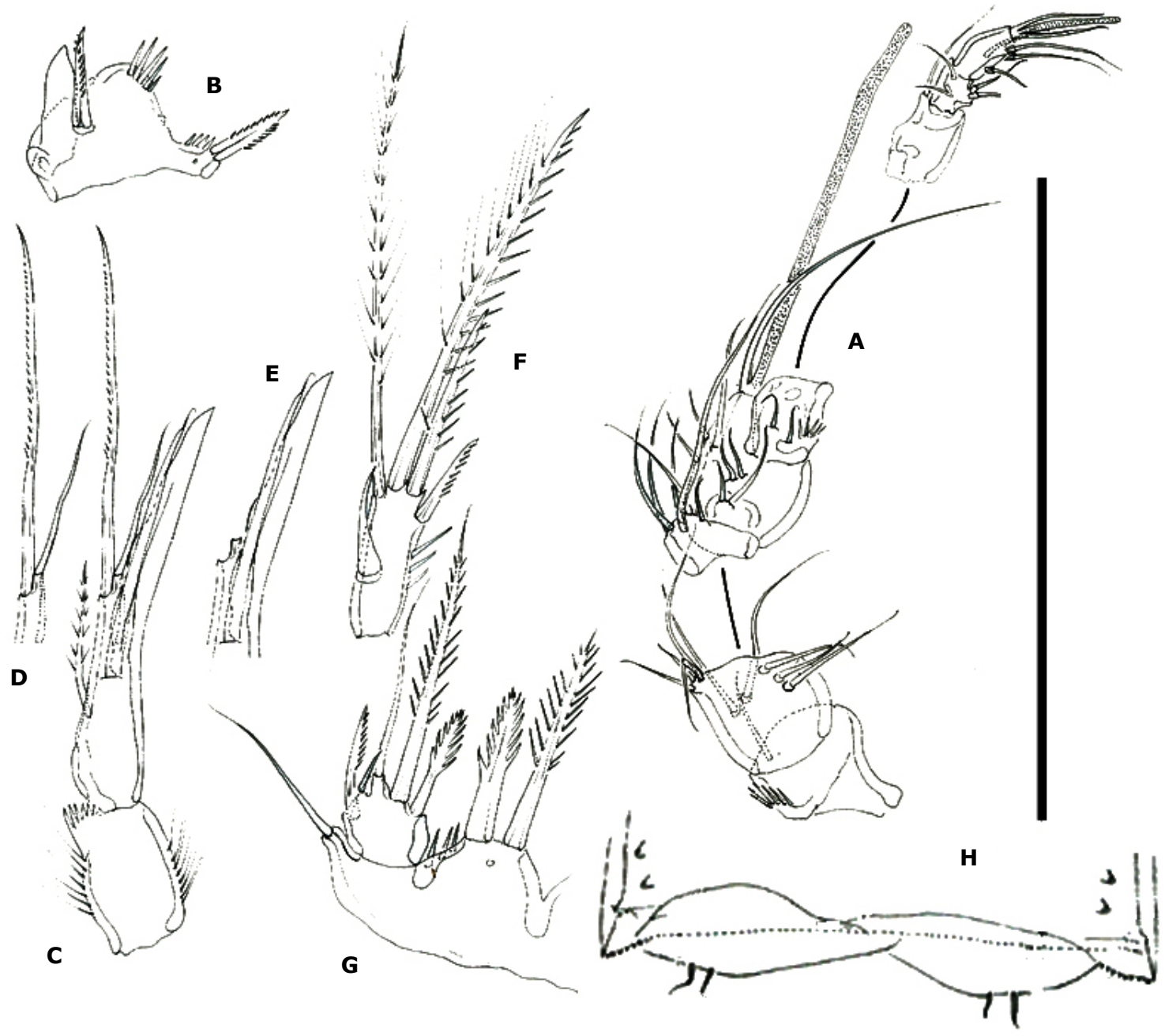

Figure 8. Schizopera evelynae sp. nov., male. A, antennule; B, basis of $\mathrm{P} 1$, anterior; $\mathrm{C}$, P2ENP, anterior; $D$, distal armature of P2ENP2; E, distal apophysis and flagellate element on P2ENP2; F, P3EXP3; G. P5, anterior; H, P6, anterior. A-H, paratype EMUCOP 0412-04. Scale bar: $100 \mu \mathrm{m}$.

P5 (Figure 8G). Exopod with 5 setae. Baseoendopod with two strong elements ornamented as figured, plus outer seta of basis.

P6 (Figure 8H). Each leg represented by one plate armed with 2 minute setae.

Variability. Female (11 females analyzed). The left P1ENP 2 of one female was observed to possess one instead of two setae; the left P1ENP 1 of one female lacks the lateral seta. The left P5ENP of two females possess five instead of four setae. Male (11 males analyzed). The right P2ENP 1 of one male was observed to possess a big spine instead of spinules.

Distribution and ecology. Schizopera evelynae sp. nov. is currently known from a single locality
P5 (Figura 8G). Exópodo con 5 setas. Basoendópodo con dos elementos fuertes ornamentados como se ilustra, más seta externa de la base.

P6 (Figura 8H). Cada pata representada por una placa con 2 setas diminutas.

Variabilidad. Hembra (se analizaron 11 hembras). EI P1ENP 2 izquierdo de una hembra, con 1 en vez de 2 setas; el P1ENP 1 izquierdo de una hembra carece de seta lateral. El P5ENP izquierdo de dos hembras posee 5 en lugar de 4 setas. Machos (se analizaron 11 machos). Se observó que el P2ENP 1 derecho de 1 macho posee una espina grande en lugar de espínulas.

Distribución y ecología. Schizopera evelynae sp. nov. se conoce actualmente de un sitio solamente, el 
only, the protected coastal system Laguna Navío Quebrado, on the Caribbean coast of Colombia. This large (surface area of $10.7 \mathrm{~km}^{2}$ ) lagoon is a shallow water body (depth 0.3-1.1 m), whose temperature varies over the seasons from 28 to $31^{\circ} \mathrm{C} ; \mathrm{pH}$ values during sampling ranged between 7.8 and 8.3. In the surveyed area $S$. evelynae $\mathrm{sp}$ nov. was recorded in both the limnetic region and the vegetation zones (mangroves), being more frequent in the former habitat where salinity was highest (28 PSU). Fourteen species of Harpacticoida were reported in this water body (28).

\section{DISCUSSION}

Among the species of the genus Schizopera, $S$. evelynae sp. nov. seems to be closely related to S. giselae (16) and to S. pratensis (28) given the armature formula of P1-P4. However $S$. evelynae sp. nov. can be separated from $S$. giselae and $S$. pratensis by the relative length of P1Enp 1 (P1ENP 1 being longer that the exopod in $S$. evelynae, but as long as exopod in S. pratensis and S. gisea/e); length/width ratio of P1ENP 2 (about 1.4 in $S$. evelynae sp. nov. and 2.4 and 3 in $S$. giselae and $S$. pratensis, respectively). The anal operculum of $S$. evelynae sp. nov. is similar to that of $S$. pratensis, but differs from that of $S$. giselae in the setular ornamentation (with two rows of setules in S. giselae, but smooth in S. evelynae sp. nov. and $S$. pratensis). Also, the outer proximal and distal spines on the P4EXP 3 are different in length in $S$. evelynae sp. nov., and $S$. pratensis, but these outer spines are of the same length in S. giselae. The female P5 of $S$. evelynae sp. nov. is similar to that of $S$. giseale, but differs from that of $S$. pratensis in the shape of the exopod and in the relative length of the exopodal setae (exopod rounded in $S$. evelynae sp. nov. and $S$. giselae, but comparatively more elongate in $S$. pratensis).

The male of $S$. evelynae sp. nov. differs from the male of $S$ pratensis in the length/width ration of P2ENP 2 (about 2.7 times as long as wide in $S$. evelynae sp. nov., but as long as wide in S. pratensis), in the shape of the apical elements on P2ENP 2 (one straight spine and three slender elements in $S$. evelynae sp. nov. but two long curved spines and one slender element in S. pratensis), in the length/width ration of the caudal rami (as long as wide in S. evelynae sp. nov., but 1.3 times as long as wide in $S$. pratensis), in the baseoendopodal lobe:exopod length ratio (longer than the exopod in $S$. evelynae sp. nov., but both rami of about the same length in S. pratensis). sistema costero protegido de Laguna Navío Quebrado, en la Costa Caribe de Colombia. Este gran sistema lagunar (superficie de $10.7 \mathrm{~km}^{2}$ ) es un cuerpo de agua poco profundo (profundidad entre 0.3-1.1 m), cuya temperatura varia a estacionalmente entre $28-31^{\circ} \mathrm{C}$; los valores de $\mathrm{pH}$ durante el muestreo estuvieron entre 7.8 y 8.3. En el área explorada se registró a $S$. evelynae sp. nov., tanto en la región limnética como en las zonas de vegetación (manglares), siendo más frecuentes en el primer hábitat donde la salinidad era mayor (28 PSU). Se reportaron 14 especies de Harpacticoida en este cuerpo de agua (28).

\section{DISCUSIÓN}

Entre las especies del género Schizopera, S. evelynae sp. nov. parece estar muy relacionada con S. giselae (16) y S. pratensis (28) dada la fórmula de setas y espinas de P1-P4. Sin embargo, S. evelynae sp. nov. se puede separar de $S$. giselae y $S$. pratensis por el tamaño relativo del P1Enp 1 (P1ENP 1 más largo que el exópodo en $S$. evelynae, pero tan largo como el exópodo en $S$. pratensis y $S$. giseale); la relación de largo/ancho de P1ENP 2 (cerca de 1.4 en $S$. evelynae sp. nov. y 2.4 y 3 en $S$. giselae y $S$. pratensis, respectivamente). El opérculo anal de S. evelynae sp. nov. es similar al de S. pratensis, pero difiere del de $S$. giselae en la ornamentación setular (con dos filas de pequeñas sétulas en S. giselae, pero liso en $S$. evelynae sp. nov. y S. pratensis). Así mismo, las espinas externas proximales y distales en el P4EXP 3 son diferentes en tamaño en $S$. evelynae sp. nov. y S. pratensis, pero estas espinas externas son del mismo tamaño en S. giselae. La P5 de la hembra de $S$. evelynae sp. nov. es similar a la de $S$. giseale, pero difiere de la de $S$. pratensis en la forma del exópodo y en el tamaño relativo de la setas del exópodo (exópodo redondo en $S$. evelynae sp. nov. y S. giselae, pero comparativamente más alargado en $S$. pratensis).

El macho de S. evelynae sp. nov. difiere del macho de $S$. pratensis en la relación largo/ancho del P2ENP 2 (cerca de 2.7 veces más largo que ancho en $S$. evelynae sp. nov., pero tan largo como ancho en $S$. pratensis), en la forma de los elementos apicales del P2ENP 2 (1 espina recta y 3 elementos delgados en $S$. evelynae sp. nov., pero dos espinas curvas y un elementos delgado en S. pratensis), en la relación largo/ancho de las ramas caudales (tan largas como anchas en S. evelynae sp. nov., pero 1.3 veces más largas que anchas en $S$. pratensis), en la relación de la longitud del lóbulo del basoendópodo:exópodo (más largo que el exópodo en $S$. evelynae sp. nov., pero ambos de la misma longitud en $S$. pratensis) . 
Unfortunately, the male of $S$. giselae remains unknown and cannot be compared with the male of $S$. evelynae sp. nov.

In the following section, an identification key that might prove useful, at least, for preliminary identification of the valid Schizopera species for America, is presented. The key is based on female characters, because the male of S. giselae, S. triacantha, S. californica are unknown.
Desafortunadamente, el macho de S. giselae no se conoce y no puede ser comparado con el macho de S. evelynae sp. nov.

En la siguiente sección se presenta una clave de identificación de las especies validas de Schizopera de América que puede resultar útil, por lo menos, para una identificación preliminar. La clave está basada en caracteres de la hembra, debido a que los machos de S. giselae, S. triacantha y $S$. californica no se conocen.

\section{Key to america species of the genus schizopera.}

1A. ENP P1 2-segmented

1B. ENP P1 3-segmented

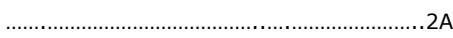

2A. P4 ENP 2-segmented

Schizopera gauldi (9)

3A. anal operculum with two rows of setules, outer spines of third exopodal segment of P4 equal in length .. giseale(16)

3B. anal operculum smooth, Outer spines of third exopodal segment of $\mathrm{P} 4$ unequal in length

S. evelynae sp. nov

4A. A2 EXP 1-segmented

4B. A2 EXP 2 or 3-segmented

5A. P2ENP2 with two inner setae, length/width ratio of caudal rami about 2.5

$5 B$. P2ENP2 with one inner seta, length/width ratio of caudal rami about 1

S. anomala(13)

6A. A2 EXP 3-segmented

carolinensis (13)

6B. A2 EXP 2-segmented

chiloensis (16)

7A. P3 EXP3 with 5 setae

7B. P3 EXP3 with 4 setae

8A. P5 ENP with 3 setae

8B. P5 ENP with 5 setae

9A. P3 ENP 3 with 3 setae

9B. P3 ENP 3 with 4 setae

10A. P3 and P4 ENP 1 without inner seta

10B. P3 and P4 ENP1 with one inner seta

11A. Caudal rami less than 2 times as long as wide

11B. Caudal rami about 2 or 2.5 times as long as wide

12A. P3-P4EXP2 without inner setae

12B. P3-P4EXP2 with inner setae

13A. length/width ratio of P1ENP 1 about 2.5

13B. length/width ratio of P1ENP 1 more than 2.5

14A. caudal rami with inner setules; length/width ratio of P1ENP 1 about 4.3

14B. caudal rami without inner setules; length/width ratio of P1ENP 1 less than 4.3

$15 \mathrm{~A}$. outer spine of the last endopodal segment of P1 thick; length/width ratio of P1ENP3 about 2

15B. outer spine of the last endopodal segment of P1 slender; length/width ratio of P1ENP 3 about 2.5-3

$16 \mathrm{~A}$. inner spine of the basis of P1 short; length/width ratio of P1ENP 3 about 3

16B. inner spine of the basis of P1 long; length/width ratio of P1ENP 3 about 2.5

17A. Caudal rami about 2 times as long as wide; P1ENP1 slightly shorter than EXP
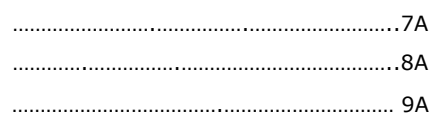

S. haitiana (7)

S. triacantha (7)

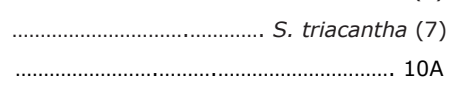

S. borutzkyi (12)

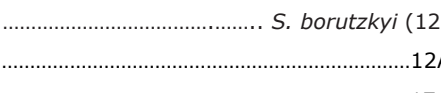

S. costaricana (19) 


\section{REFERENCES}

1. Hendrickx ME, Fiers F. Copépodos Harpacticoida asociados con crustáceos decápodos. Cienc Mar 2010; 14:3-30.

2. Diversity and Geographic Distribution of Marine Planktonic Copepods [database on the Internet]. Banyuls-sur-Mer: Observatoire Océanologique de Banyuls, Université Pierre et Marie Curie (Paris VI) - CNRS/INSU (France). c2005-2014. [cited 2012 September 29]. [about 9p.]. Available from: http:// copepodes.obs-banyuls.fr/en

3. Sars GO. An Account of the Crustacea of Norway. Volume V. Copepoda Harpacticoida, Parts 7-10. Plates XLVIII-LXXX, Bergen, Bergen Museum; 1905.

4. Boeck A. Oversigt over de ved Norges Hyster iagttagne Copepoder henhörende til Calanidernes, Cyclopidernes og Harpacticidernes Familier. Forh VidSelsk Kristiania 1865; 1864:1-57.

5. Karanovic T, Cooper S.J.B. Explosive radiation of the genus Schizopera on a small subterranean island in Western Australian (Copepoda:Harpacticoida): unraveling the cases of cryptic speciation, size differentiation and multiple invasions. Invertebr Syst 2012; 26:115-192.

6. World Register of Marine Species [database on the Internet]. Ostende: Flanders Marine Institute (Belgium). c2008. [cited 2014 January 20]. [about 18p.]. Available from: http://www.marinespecies.org/aphia.php.

7. Chappuis PA. Copepoda Harpacticoida der Deutschen Limnologischen Sunda-Expedition. Arch Hydrobiol 1931; Suppl 8:S512-584.

8. Kiefer F. Neue Ruderfusskrebse von der Insel Haiti. Zool Anz 1934; 108:227-233.

9. Herbst HV. Copepoden (Crustacea, Entomostraca) aus Nicaragua und Südperu. Gewässer und Abwässer. 1960; 27:27-54.

10. Chappuis PA. Rouch R. Un nouvelle Nitocrella de Minorque. Arch Zool Exp 1961; 99:245-247.

11. Rouch R. Harpacticoïdes (Crustacés Copépodes) d'Amerique du Sud. In: C. Delamare Deboutteville \& Rapoport E. (Ed), Biologie de I'Amerique Australe, Paris, Éditions du Centre National de la Recherche Scientifique; 1962.
12. Lang K. Copepoda Harpacticoidea from the Californian Pacific coast. Kungl. svenska VetenskapsakademiensHandlingar 1965; 10(2):1-560.

13. Montschenko V. Beitrag zur Kenntnis der Gattung Schizopera (Crustacea, Harpacticoida) im Schwarzen Meer Zool Anz 1967: 178:367-374.

14. Coull BC. Meiobenthic Harpacticoida (Crustacea, Copepoda) from the North Carolina continental shelf. Cah Biol Mar.1971; 12:195-237.

15. Petkovski TK. Subterrane SüsswasserHarpacticoida von Kuba (Vorläufige Mitteilung). Résultats des expéditions biospéologiques cubanoroumaines à Cuba, Academiei Republicii Socialiste. Bucarest; 1973.

16. Jiménez-Álvarez MP. Harpacticoid copepods from Una do Prado River (São Paulo, Brazil): genus Schizopera. Hydrobiologia 1988; 167/168:435-444.

17. Mielke W. Description of some benthic Copepoda from Chile and discussion on the relationship of Paraschizopera and Schizopera (Diosaccidae). Microfauna Marina 1992; 7:79-100.

18. Mielke W. Species of the taxon Schizopera (Copepoda) from the pacific coast of Costa Rica. Microfauna Marina 1995; 10:89-116.

19. Kunz H. Schizopera hawaiiensis sp. n. (Copepoda, Harpacticoida) aus einer Lagune auf Oahu, Hawaii-Inseln. Mitt. Hamb Zool Mus Inst 1995; 92:65-72.

20. Karanovic T. Subterranean copepods from arid Western Australia. Crustaceana Monographs 2004; 3:1-366.

21. Apostolov A. Harpacticoïdes (Crustacea, Copepoda) de la mer Égée (plages de Kavala, Grèce du nord). Hist Nat Bulg 2008; 19: 5-33.

22. Karanovic T, McRae J. The genus Schizopera (Copepoda: Harpacticoida) in the Pilbara region of Western Australia, with a description of new species and its molecular and morphological affinities. Rec Aus Mus 2013; 28:119-140. 
23. Apostolov A. Genres et sous-genres nouveaux de la famille Diosaccidae Sars et Cylindropsyllidae Sars, Lang (Copepoda, Harpacticoidea). Acta Zool Bulgar 1982; 19:37-42.

24. Wells, JBJ, Rao GC. The relationships of the genus Schizopera Sars within the family Diosaccidae (Copepoda:Harpacticoida). Zool J Linnean Soc 1976 58:79-90.

25. Bodin P. Catalogue of the new marine Harpacticoid Copepods (1997 Edition). Documents du travail de I'Institut Royal des Sciences Naturelles de Belgique. 1997.

26. Wells JBJ. An annotated checklist and keys to the species of Copepoda Harpacticoida (Crustacea). Zootaxa 2007; 1568:1-872.
27. Huys R, Boxshall GA. Copepod evolution. London: The Ray Society 1991; 113-152.

28. Fuentes-Reinés, J.M, Suárez-Morales, E. Annotated checklist and new records of Harpacticoida (Copepoda) from a coastal system of northern Colombia, South America. Crustaceana 2014; 87(2):212-255.

29. Noodt W. Schizopera pratensis n. sp. von Salwiesen der deutschen Meeresküste. Kieler Meeresforsch. 1958; 14:223-225. 